\title{
Oléagineux et protéagineux : concilier l'atténuation du changement climatique et la nécessité de s'y adapter
}

\author{
Anne Schneider ${ }^{1, *}$, Anne-Sophie Perrin ${ }^{2}$ et Cécile Le Gall ${ }^{1}$ \\ 1 Terres Inovia, centre de Grignon, avenue Lucien-Brétignières, 78850 Thiverval-Grignon, France \\ 2 Terres Inovia, c/o UMR Eco§Sols, bâtiment 12, 2, place Pierre-Viala, 34060 Montpellier, France
}

Reçu le 19 janvier 2017 - Accepté le 19 janvier 2017

Depuis plusieurs années, la France se fixe des objectifs ambitieux pour réduire les impacts environnementaux de ses productions végétales et notamment réduire leurs parts dans les émissions de gaz à effet de serre (GES) responsables de la part anthropique du changement climatique. Par ailleurs, ces dernières années, les accidents climatiques ont touché toutes les espèces des productions végétales annuelles métropolitaines entraînant la réduction des rendements et des surfaces récoltées. Ainsi, il devient urgent de changer la conception des systèmes agricoles et agroalimentaires en conciliant adaptation et atténuation du changement climatique.

L'étape de la production des matières premières agricoles étant prépondérante, répondre à ces deux enjeux d'envergure doit faire appel à une démarche basée sur l'agronomie. Si l'on veut à la fois réduire l'usage des intrants dont la part de responsabilité est conséquente pour les GES des productions végétales, et favoriser la robustesse du potentiel de production agricole face aux aléas, alors il est judicieux de mobiliser des processus inhérents aux écosystèmes pour qu'ils contribuent davantage, en plus des intrants, à la nutrition des cultures et à une meilleure régulation des pressions biotiques.

Pour mobiliser ces services écosystémiques, Terres Inovia identifie deux volets à favoriser : une plus grande fertilité des sols et une plus grande diversité fonctionnelle au sein des familles botaniques des cultures et des agencements sur l'exploitation agricole.

\section{Adaptation au changement climatique}

Face au changement climatique, les pistes d'adaptation qui sont investiguées en priorité portent sur la modulation des itinéraires techniques et l'évolution des critères d'amélioration variétale de chaque culture. Cependant, l'urgence des problèmes et l'innovation requise face à leur ampleur nécessitent de mobiliser également des changements de stratégies plus drastiques, engageant la re-conception des systèmes, d'autant plus lorsque l'on souhaite conjuguer l'adaptation avec la réduction des intrants. Terres Inovia alimente et souhaite renforcer ce type d'investigations en étroite collaboration avec

\footnotetext{
* Correspondance : a.schneider@terresinovia.fr
}

des réseaux d'agriculteurs et de conseillers. Les agriculteurs qui modulent profondément leurs systèmes de culture pour sortir d'impasses techniques ou réduire fortement leurs intrants partent souvent du double constat suivant :

- le sol, matrice clé de la production agricole, est plus qu'un simple réceptacle de nutriments et son bon état chimique, physique et biologique est garant de la productivité ;

- l'implantation de la culture doit être réussie, car c'est la base d'une culture robuste face aux différents stress à venir au cours de la campagne agricole.

C'est pourquoi les systèmes à moindres intrants requièrent de mieux appréhender les dynamiques des matières organiques et des activités biologiques des sols en interaction avec les cultures et leurs itinéraires techniques. Des premiers éléments soulignent que l'association des familles botaniques dans les systèmes de culture, l'introduction de légumineuses ou encore l'augmentation de la restitution au sol de matières organiques de différentes compositions biochimiques (notamment via les résidus de culture) tendent à favoriser la fertilité physique, chimique et un bon fonctionnement biologique des sols.

\section{Atténuation du changement climatique}

Pour agir en faveur de l'atténuation du changement climatique, Terres Inovia travaille sur deux pistes complémentaires :

- identifier les leviers prioritaires en analysant les facteurs de variabilité des émissions du protoxyde d'azote $\left(\mathrm{N}_{2} \mathrm{O}\right)$ liés aux grandes cultures (GES représentant le poste le plus important pour les émissions du secteur des grandes cultures) ;

- accompagner le développement des légumineuses (protéagineux, soja, légumineuses en couverts de service) qui représente un des dix premiers leviers techniques d'atténuation en agriculture (Pellerin et al., 2013).

De plus, le rôle des cultures sur le stock de matières organiques des sols (MOS) est un sujet à creuser pour favoriser, si possible, le rôle de puits potentiel de carbone du sol. 


\section{Comprendre les facteurs de variation des émissions de $\mathrm{N}_{2} \mathrm{O}$ liés aux grandes cultures pour agir}

En partenariat avec l'Inra-Ecosys, Terres Inovia a développé des compétences sur les processus, les méthodes de suivi et les outils de prédiction relatifs aux émissions de $\mathrm{N}_{2} \mathrm{O}$. Dans le cadre de l'UMT GES- $\mathrm{N}_{2} \mathrm{O}$ que portait l'institut (en tant que Cetiom à l'époque) en partenariat avec l'Inra-Ecosys à Grignon, des mesures expérimentales ont été compilées sur le territoire français sous céréales et oléoprotéagineux. En parallèle, une nouvelle méthode d'estimation des émissions de $\mathrm{N}_{2} \mathrm{O}$ produites par les sols cultivés a été travaillée : à partir des données expérimentales collectées, un modèle statistique a été mis au point, qui propose de retenir, comme facteurs majoritaires, les quantités d'azote minéral et organique, le pH du sol et la pluviométrie (Le Gall et al., 2014).

Pour viser une réduction des risques d'émissions de $\mathrm{N}_{2} \mathrm{O}$ sur le colza, un volet GES a été renforcé depuis 2011, en plus de la performance énergétique, au sein de la démarche de progrès des bilans environnementaux du biodiesel, impliquant une cinquantaine d'organismes stockeurs. La meilleure gestion de la nutrition azotée est la clé principale d'action pour le colza (voir article dans ce dossier de Simonin, 2017).

\section{Le levier de l'azote symbiotique des légumineuses pour réduire les GES}

En lien avec d'autres partenaires comme notamment l'Inra-Grignon au sein de l'UMT Alter'N, Terres Inovia s'investit pour étudier comment favoriser et gérer au mieux l'entrée d'azote symbiotique dans les systèmes, car cette source alternative permet de réduire l'usage des engrais minéraux. Vu l'absence d'apport d'engrais azotés sur les cultures de légumineuses et la réduction des engrais apportés sur la culture suivante, et vu l'absence d'émission de $\mathrm{N}_{2} \mathrm{O}$ spécifiquement liée au processus biologique de fixation symbiotique, les légumineuses permettent d'éviter une série d'impacts environnementaux liés à la production et à l'épandage de ces engrais (Cellier et al., 2015). Des mesures pendant trois campagnes (2008-2011) ont montré que les émissions de $\mathrm{N}_{2} \mathrm{O}$ sous pois protéagineux étaient en moyenne significativement inférieures à celles de cultures fertilisées (blé ou colza) en conduite mono-spécifique en conditions françaises (Jeuffroy et al., 2013) et équivalente à celles sous blé non fertilisé. Par ailleurs, en plus des services écosystémiques liés à l'azote, les légumineuses rendent d'autres services écosystémiques aux agrosystèmes (Schneider et Huyghe, 2015), et notamment via la fertilité des sols, même si les mécanismes mobilisés sont encore à creuser.

\section{Les associations de familles botaniques et la fertilité physique, chimique et biologique des sols}

Les associations de culture entre légumineuses et graminées permettent de valoriser la complémentarité des familles botaniques à explorer des stratégies de nutrition azotée spécifiques. La fixation symbiotique est alors renforcée pour l'acquisition d'azote par les légumineuses alors que le système racinaire des graminées exploite seul l'azote minéral du sol en explorant plus largement les horizons. La structure et le fonctionnement biologique du sol sont favorables à une meilleure robustesse des plantes, car les rendements des associations sont à la fois plus élevés et plus stables que les mêmes espèces en culture mono-spécifique sur des surfaces équivalentes (Corre-Hellou et al., 2013).

L'utilisation de couverts gélifs de légumineuses sous colza est une pratique qui confirme son intérêt depuis 2011 dans certains contextes pédoclimatiques (Cadoux et al., 2015). Sa maitrise permet de conforter l'implantation du colza, de réduire de $30 \mathrm{~kg}$ l'azote apporté, tout en cumulant le maintien $\mathrm{du}$ rendement, voire son augmentation (de $10 \%$ ou plus en conditions difficiles), avec même une réduction possible des herbicides et insecticides, avec des baisses des attaques d'altises et de charançons du bourgeon terminal. Cette association de couverts de légumineuses avec le colza est tout spécialement intéressante en sols superficiels. Conjuguée avec l'insertion de protéagineux dans la succession culturale, elle s'avère bénéfique pour la fertilité des sols dans les réseaux d'agriculteurs l'ayant adoptée, et donc pour la robustesse des performances des systèmes de cultures malgré des aléas plus extrêmes lors de la campagne agricole.

\section{La dynamique des matières organiques et des activités biologiques dans les sols encore trop peu connue}

Les paramètres physiques, chimiques, biologiques et organiques des sols sont fortement impliqués dans l'émission ou non de $\mathrm{N}_{2} \mathrm{O}$, dont les productions végétales sont majoritairement responsables. Or l'état de ces paramètres est aussi crucial pour la fertilité des sols et donc la productivité agricole. Les MOS, composées à environ $58 \%$ de carbone organique, permettent le bon fonctionnement et la durabilité des agrosystèmes. Les MOS assurent le stockage ou la mise à disposition (lorsqu'elles sont minéralisées) des éléments nutritifs dont les plantes ont besoin, elles stimulent l'activité biologique (dont elles sont la source d'énergie et de nutriments), et elles jouent également un rôle majeur sur les caractéristiques physiques du sol: stabilité vis-à-vis des pluies et des phénomènes de tassement, aération, capacité de rétention en eau.

S'il n'y a pas encore de relation statistique établie entre l'activité biologique des sols et la productivité agricole, les présomptions sont fortes et des liens existent pour certaines fonctions des sols. Les micro-organismes, les nématodes et la macrofaune sont des acteurs clés du fonctionnement des sols à l'origine de nombreux services écosystémiques (notamment Altieri, 1999). Ainsi, la biodiversité dans les sols modifie le cycle des matières organiques et les compétitions entre micro-, mésoet macro-organismes du sol qui temporisent les infections issues du sol, les processus de dénitrification et donc d'émissions de $\mathrm{N}_{2} \mathrm{O}$, la structure du sol et les fuites en nitrate.

Il apparaît crucial d'investiguer plus amplement la dynamique et le rôle des composantes biologiques et des MOS dans les mécanismes de résilience qui permettent à la production agricole de maintenir sa multi-performance face aux perturbations subies par les milieux. 


\section{Conclusion}

En conclusion, pour les oléagineux et protéagineux, comme pour toutes les grandes cultures, l'enjeu se situe au niveau de la stratégie de conception et de gestion de systèmes de production qui puissent favoriser à la fois réduction des GES et des autres émissions potentiellement polluantes, tout en mobilisant un ensemble de services écosystémiques autorisant la réduction des intrants et favorables à une meilleure stabilité des performances agronomiques et économiques.

\section{Références}

Altieri MA. 1999. The ecological role of biodiversity in agroecosystems. Agric Ecosyst Environ 74: 19-31.

Cadoux S, Sauzet G, Valantin-Morison M, et al. 2015. Intercropping frost-sensitive legume crops with winter oilseed rape reduces weed competition, insect damage, and improves nitrogen use efficiency. OCL 22(3): D302.

Cellier P, Schneider A, Thiébeau P, Vertès F. 2015. Impacts environnementaux de l'introduction de légumineuses dans les systèmes de production. In : Schneider A, Huyghe C, eds. Les légumineuses pour des systèmes agricoles et alimentaires durables. Versailles : Éditions Quae, p. 339-413.

Corre-Hellou G, Bédoussac L, Bousseau D, et al. 2013. Associations céréale-légumineuse multi-services. Innov Agron 30: 41-57.

Jeuffroy MH, Baranger E, Carrouée B, et al. 2013. Nitrous oxide emissions from crop rotations including wheat, oilseed rape and dry peas. Biogeosciences 10(3): 1787-1797. doi: 10.5194/bg10-1787-2013.

Le Gall C, Jeuffroy MH, Hénault C, et al. 2014. Analyser et estimer les émissions de $\mathrm{N} 2 \mathrm{O}$ dans les systèmes de grandes cultures français. Innov Agron 12: 97-112.

Pellerin S, Bamiere L, Angers D, et al. 2013. Quelle contribution de l'agriculture française à la réduction des émissions de gaz à effet de serre ? Potentiel d'atténuation et coût de dix actions techniques. In : Synthèse d'étude, Inra, $92 \mathrm{p}$.

Schneider A, Huyghe C. 2015. Les légumineuses pour des systèmes agricoles et alimentaires durables. Versailles : Éditions Quae, $473 \mathrm{p}$.

Simonin P. 2017. Observation des pratiques de fertilisation azotée par un outil d'aide à la décision pour le colza. OCL. doi: 10.1051/ocl/ 2016056.

Citation de l'article : Schneider A, Perrin A-S, Le Gall C. 2017. Oléagineux et protéagineux : concilier l'atténuation du changement climatique et la nécessité de s'y adapter. $O C L, 2017,24(1) \mathrm{D} 101$. 